\title{
Biotransformation of congo red in a UASB reactor under salinity conditions using immobilized redox mediator in granular activated carbon
}

\section{Biotransformación de rojo congo en un reactor UASB bajo condiciones de salinidad utilizando mediadores redox inmovilizados en carbón activado granular}

Glenda A. Espinoza1, Verónica Almaguer-Cantú1, Refugio B. García-Reyes², Edna R. Meza ${ }^{3}$, Denisse Serrano-Palacios ${ }^{3}$, Luis H. Alvarez-Valencia ${ }^{*}$

${ }^{1}$ Facultad de Ciencias Biológicas, Universidad Autónoma de Nuevo León (UANL). Av. Universidad S/N, Cd. Universitaria, C.P. 66455, San Nicolás de los Garza, Nuevo León, Mexico.

2 Facultad de Ciencias Químicas, Universidad Autónoma de Nuevo León (UANL). Av. Universidad S/N, Cd. Universitaria, C.P. 66455, San Nicolás de los Garza, Nuevo León, Mexico.

${ }^{3}$ Departamento de Ciencias del Agua y Medio Ambiente, Instituto Tecnológico de Sonora (ITSON). 5 de febrero 818 Sur, C.P. 85000, Ciudad Obregón, Sonora, Mexico.

${ }^{4}$ Departamento de Ciencias Agronómicas y Veterinarias, Instituto Tecnológico de Sonora (ITSON). 5 de febrero 818 Sur, C.P. 85000, Ciudad Obregón, Sonora, Mexico.

${ }^{*}$ Corresponding author

E-mail address: luis.alvarez@itson.edu.mx (L. H. Alvarez-Valencia).

Article history:

Received: 8 June 2021 / Received in revised form: 19 August 2021 / Accepted: / 21 August 2021 / Published online: 1 October 2021. https://doi.org/10.29267/mxib.2021.6.4.17

\section{ABSTRACT}

Azo dyes are susceptible to be treated by reductive biotransformation process under anaerobic conditions. The process can be accelerated by the addition of 
quinones and humic substances acting as redox mediators (RM). In this study, the anthraquinone-2-sulfonate (AQS) was immobilized on granular activated carbon (GAC) to evaluate the reductive biotransformation of congo red (CR) in an up-flow anaerobic sludge blanket reactor (UASB). The syudy was divided in five stages, where the reactors with immobilized RM and without RM were operated under different salinity levels (1\% and $3 \%$ ) and hydraulic retention times (HRT $=5$ and 10 h). The reactor with immobilized RM (GAC-AQS) achieved a decolorization efficiency of $96.1 \%$ and substrate consumption of $98.8 \%$ with a HRT $=15 \mathrm{~h}$ and $1 \%$ of salinity. Nonetheless, with a salinity of $3 \%$ and the same HRT, the efficiency was similar (95.6\%). The reactor provided with unmodified GAC achieved values below those observed in the reactor GAC-AQS, with decolorization efficiencies of $90.8 \%$ and $75.8 \%$, and substrate consumption of $97.1 \%$ and $88.4 \%$, for the stages IV and V, respectively. The microbial consortium sued was able to promote the biotransformation of azo dye and no inhibitory effects were identified.

Keywords: anaerobic consortium, electrophilic contaminant, reductive decolorization, textile effluents.

\section{RESUMEN}

Los colorantes azo son susceptibles de ser tratados mediante procesos de biotransformación reductiva bajo condiciones anaerobias, que pueden ser acelerado con la adición quinonas y ácidos húmicos que actúan como mediadores redox (MR). En este trabajo se inmovilizó antraquinona-2-sulfonato (AQS) en carbón activado granular (CAG) para evaluar la biotransformación reductiva de rojo congo $(R C)$ en un reactor anaerobio de flujo ascendente (UASB). El estudio se dividió en cinco etapas, en donde los reactores con MR inmovilizados y sin MR fueron operados con distintas concentraciones salinidad (1\% y $3 \%)$ y tiempos de residencia hidráulico (TRH = 5 y $10 \mathrm{~h}$ ). El reactor con MR inmovilizados (CAGAQS) alcanzó una eficiencia de decoloración de $96.1 \%$ y de consumo de sustrato de $98.8 \%$ con el $\mathrm{TRH}=15 \mathrm{~h}$ y $1 \%$ de salinidad. Con $3 \%$ de salinidad y el mismo $\mathrm{TRH}$, la eficiencia de decoloración fue similar (95.6\%). El reactor con CAG no modificado alcanzó valores por debajo del reactor CAG-AQS, con eficiencias de decoloración de $90.8 \%$ y $75.8 \%$, y de consumo de sustrato de $97.1 \%$ y $88.4 \%$, para las etapas IV y V, respectivamente. El consorcio microbiano utilizado llevó a cabo la biotransformación del colorante y no presentó efectos inhibitorios.

Palabras clave: contaminante electrofílico, consorcio anaerobio, decoloración reductiva, efluentes textiles. 


\section{INTRODUCCIÓN}

La industria textil utiliza grandes volúmenes de agua para realizar el teñido de fibras y telas. Como consecuencia, se genera agua residual que se caracteriza por presentar importantes variaciones en sus parámetros, como la demanda química de oxígeno, demanda bioquímica de oxígeno (DBO), $\mathrm{pH}$, color y salinidad (3\% a $20 \%$ ). Además, los componentes específicos también varían de forma importante, ya que contiene distintos compuestos orgánicos, colorantes y químicos empleados en las diferentes etapas del teñido (Talarposhti et al., 2001; Fernando et al., 2013). La obtención de $1 \mathrm{~kg}$ de producto textil puede generar la descarga de 100 a $200 \mathrm{~L}$ de aguas residuales con altas concentraciones de colorantes (Xiao \& Roberts, 2010), debido a que durante el proceso de teñido no se aprovecha hasta el $50 \%$ del colorante, debido a que no se fijan al tejido (O'Neill et al., 1999). Por tal motivo, se reportan concentraciones de colorantes de $100-500 \mathrm{mg} / \mathrm{L}$ en las aguas residuales (Isik, 2004). La gran mayoría (60-70\%) de los colorantes utilizados en la industria textil son colorantes azo, cuya característica principal es el enlace insaturado de dos moléculas de nitrógeno (enlace azo, $\mathrm{N}=\mathrm{N}$ ) que unen estructuras aromáticas sustituidas (Van Der Zee \& Cervantes, 2009; Martínez et al., 2018).

Los colorantes azo son contaminantes electrofílicos, lo que los hace difíciles de ser tratados efectivamente por procesos aerobios convencionales, pero son factibles de someterse a procesos de biotransformación reductiva bajo condiciones anaerobias (Brown \& Laboureur,1983; Pagga \& Brown, 1986). Sin embargo, debido a la complejidad estructural y gran resistencia a ser degradados, muchos compuestos electrofílicos recalcitrantes pueden generar efectos inhibitorios sobre los consorcios anaerobios. Además, los efluentes textiles también contienen altas concentraciones de salinidad, que también contribuye a inhibir los consorcios microbianos. Desde hace dos décadas, quinonas modelo o humus han sido aplicados como mediadores redox (MR) en procesos de biotransformación reductiva de este tipo de contaminantes. Los MR aceleran la tasa de reacción al mejorar la transferencia de electrones desde los donadores hasta los aceptores finales de electrones (Van Der Zee \& Cervantes, 2009). El empleo de quinonas modelo, tales como antraquinona-2-sulfonato (AQS) y antraquinona-2,6-disulfonato (AQDS) son dos de las más utilizadas en estudios de laboratorio (Martínez et al., 2013).

A pesar de las ventajas que presenta utilizar MR en procesos de decoloración, su aplicación a escala industrial tiene algunas limitaciones. Las quinonas y humus son compuestos solubles, esto implica que deben ser añadidos continuamente, lo que impactaría el costo de tratamiento y se generaría un efluente con carga orgánica. Para evitar la dosificación continua, desde hace poco más de una década se han desarrollado diferentes estrategias y materiales que permiten inmovilizar MR. Entre los materiales usados y que tienen la capacidad de actuar como MR debido que contienen grupos redox funcionales están el carbón activado granular (CAG) (Van Der Zee et al., 2003; Pereira et al., 2010), fibras de carbón 
activado (Amezquita et al., 2016), humina (Zhang et al., 2014) y planta de henna (Huang et al., 2015). La funcionalización de materiales incluye estrategias como intercambio iónico con resinas (Cervantes et al., 2010; Cervantes et al., 2011), electropolimerización en fieltro de carbón activado (Li et al., 2008; Li et al., 2009), encapsulación en alginato de calcio (Rodríguez et al., 2019), adsorción en óxidos metálicos (Alvarez et al., 2010; Alvarez et al., 2012) y a través de enlaces covalentes en CAG (Alvarez et al., 2017a), tela de carbón activado (Castañón et al., 2019) y espuma de poliuretano (Lu et al., 2010). La mayoría de los estudios con MR inmovilizados se han realizado en condiciones libres de salinidad, aspecto de suma importancia debido a las concentraciones presentes en los efluentes textiles, ya que puede causar plasmólisis y pérdida de la actividad celular, así como un deficiente consumo de sustrato en tratamientos anaerobios, influyendo en la velocidad de eliminación de colorantes azo (Xiao \& Roberts, 2010). En un estudio previo se alcanzaron eficiencias de eliminación de 40-50\% de colorantes azo bajo condiciones salinas (Guo et al., 2008), sin embargo, estas eficiencias son bajas considerando la cantidad de colorante descargado. La información sobre consorcios bacterianos que podrían tolerar altas concentraciones de salinidad y ser capaces de eliminar colorantes satisfactoriamente bajo condiciones anaerobias es escasa. Por lo tanto, se considera que la presencia de un consorcio bacteriano y la incorporación de un MR inmovilizado representa una ventaja para acelerar el proceso de degradación de colorantes en un sistema anaerobio.

El objetivo de este trabajo es evaluar el proceso de biotransformación del colorante azo rojo congo $(\mathrm{RC})$ en un reactor anaerobio de flujo ascendente (UASB) provisto de AQS inmovilizada en CAG y bajo condiciones de salinidad, con la finalidad de proponer un proceso eficiente para el tratamiento de agua residual textil.

\section{MATERIALES Y MÉTODOS}

\subsection{Reactivos e inóculo}

El medio de soporte utilizado para inmovilizar la AQS fue CAG (Carboactive 8X30, Clarimex) con un tamaño de partículas entre 0.5 y $0.8 \mathrm{~mm}$. El colorante RC y la AQS tienen una pureza de $85 \%$ (Sigma Aldrich). El lodo granular anaerobio se obtuvo de un reactor UASB instalado en una industria cervecera ubicada en Cd. Obregón, Sonora, México. El lodo fue previamente caracterizado, encontrando que su contenido de sólidos suspendidos volátiles (SSV) es $9.5 \%$ en base húmeda. El lodo fue sometido a un proceso de activación previo al experimento de decoloración de RC, utilizando un reactor UASB que fue alimentado con $1 \mathrm{~g} / \mathrm{L}$ de glucosa como fuente de energía. El periodo de activación se mantuvo hasta que el lodo alcanzó el consumo máximo de sustrato. El medio basal utilizado para la activación del lodo anaerobio granular y con el que fueron operados los reactores durante la decoloración de RC se preparó de acuerdo a la siguiente composición 
(g/L): $\mathrm{NaHCO}_{3}(3), \mathrm{NH}_{4} \mathrm{Cl}(0.3), \mathrm{KH}_{2} \mathrm{PO}_{4}(0.2), \mathrm{MgCl}_{2} \cdot 6 \mathrm{H}_{2} \mathrm{O}$ (0.03), $\mathrm{CaCl}_{2}(0.1)$ y 1 $\mathrm{mL} / \mathrm{L}$ de una solución de elementos traza constituida por $(\mathrm{g} / \mathrm{L})$ : $\mathrm{FeCl}_{2} \cdot 4 \mathrm{H}_{2} \mathrm{O}(2)$, $\mathrm{H}_{3} \mathrm{BO}_{3} \quad(0.05), \quad \mathrm{ZnCl}_{2} \quad(0.05), \quad \mathrm{CuCl}_{2} \cdot 2 \mathrm{H}_{2} \mathrm{O} \quad(0.038), \quad \mathrm{MnCl}_{2} \cdot 4 \mathrm{H}_{2} \mathrm{O} \quad(0.5)$, $\left(\mathrm{NH}_{4}\right) 6 \mathrm{Mo}_{7} \mathrm{O}_{24} \cdot 4 \mathrm{H}_{2} \mathrm{O}$ (0.05), $\mathrm{AlCl}_{3} \cdot 6 \mathrm{H}_{2} \mathrm{O}$ (0.09), $\mathrm{CoCl}_{2} \cdot 6 \mathrm{H}_{2} \mathrm{O}$ (2), $\mathrm{NiCl}_{2} \cdot 6 \mathrm{H}_{2} \mathrm{O}$ (0.092), $\mathrm{Na}_{2} \mathrm{SeO} \cdot 5 \mathrm{H}_{2} \mathrm{O}(0.162)$, EDTA (1), y $1 \mathrm{~mL} / \mathrm{L}$ de $\mathrm{HCl}(36 \%)$. El pH del medio se ajustó a 7.

\subsection{Inmovilización de AQS}

Inicialmente las partículas de CAG fueron expuestas a una solución de $250 \mathrm{~g} / \mathrm{L}$ de $\mathrm{ZnCl}_{2}$ disuelto en $\mathrm{HCl}$ concentrado (reactivo de Lucas), en donde se promueve la adición de grupos cloruro en la superficie del material. Este procedimiento consistió en agregar $3.7 \mathrm{~g}$ de CAG a $20 \mathrm{~mL}$ del reactivo de Lucas en un matraz Erlenmeyer. Se utilizó un baño de agua fría $\left(0-5^{\circ} \mathrm{C}\right)$ al inicio debido a la reacción exotérmica que se produce, y entonces se permitió reaccionar durante $24 \mathrm{~h}$ a temperatura ambiente. Posteriormente, las partículas de CAG (CAG-Cl) se lavaron tres veces con $\mathrm{HCl}$ concentrado para eliminar los residuos. Despues, las partículas CAG-Cl fueron sumergidas en $500 \mathrm{~mL}$ de una solución de AQS $4.0 \mathrm{mM}(\mathrm{pH} 7)$, permitiendo reaccionar durante $72 \mathrm{~h}$ en agitación a $150 \mathrm{rpm}$. La solución se decantó y las partículas resultantes, denominadas CAG-AQS, se secaron a $100^{\circ} \mathrm{C}$ durante $2 \mathrm{~h}$ para promover una mayor interacción. Antes y después de exponer el CAG a la solución de AQS se tomaron alícuotas de $1 \mathrm{~mL}$ para medir la concentración inicial y final en el espectrofotómetro a $330 \mathrm{~nm}$. Esto permitió determinar la capacidad de adsorción mediante un balance de masa, empleando la siguiente ecuación:

$$
q=\frac{\left(C_{0}-C_{e}\right) V}{m}
$$

donde $q$ es la capacidad de adsorción $(\mathrm{mg} / \mathrm{g}), C_{0}$ y $C_{e}$ son las concentraciones de AQS inicial y en equilibrio ( $\mathrm{mmol} / \mathrm{L}$ ), respectivamente; $V$ es el volumen $(\mathrm{L})$ de la solución de AQS y $m$ es la masa ( $\mathrm{g}$ ) de CAG. Finalmente, se utilizaron $0.5 \mathrm{~L}$ del medio basal descrito previamente para lavar a las partículas de CAG-AQS. Cada procedimiento consecutivo de lavado se puso en agitación a $150 \mathrm{rpm}$ a $30^{\circ} \mathrm{C}$ durante $24 \mathrm{~h}$. La capacidad de adsorción que resultó después de cada ciclo de lavado se calculó en base a la siguiente ecuación:

$$
q_{d}=q_{e}-\frac{C_{e} V}{m}
$$

donde $q_{e}$ es el valor inicial de capacidad de adsorción y $q_{d}$ es el valor de la capacidad de adsorción resultante de cada ciclo de lavado. 


\subsection{Operación de reactores UASB}

Se utilizaron dos columnas de vidrio como reactores UASB con una altura total de $28 \mathrm{~cm}$ y diámetro de interno de $3.2 \mathrm{~cm}$. La entrada de la alimentación del reactor es cónica ( $5 \mathrm{~cm}$ de alto) hasta alcanzar el diámetro interno. La altura de la salida para el efluente tratado es de $22 \mathrm{~cm}$, que genera un volumen de trabajo de $\sim 190$ $\mathrm{mL}$. Ambos reactores fueron inoculados con $5 \mathrm{~g}$ de SSV/L del lodo anaerobio previamente activado y se agregó $1.5 \mathrm{~g}$ de CAG-AQS (reactor CAG-AQS), equivalente a $4.0 \mathrm{mM}$ de AQS y $1.5 \mathrm{~g}$ de CAG (reactor CAG, control). En ambos casos la concentración de CAG en los reactores fue de $7.9 \mathrm{~g} / \mathrm{L}$. Además, los reactores fueron alimentados con $100 \mathrm{mg} \mathrm{RC/L}$ y $1 \mathrm{~g} / \mathrm{L}$ de glucosa, con el medio basal previamente. Se tuvieron tiempos de residencia hidráulica (TRH) de $15 \mathrm{~h}$ para las etapas I, II, IV y V, en tanto para la etapa III el TRH fue de $5 \mathrm{~h}$, lo que establece cargas orgánicas de 1.6 y $4.8 \mathrm{~kg} / \mathrm{m}^{3}$. día, respectivamente. Todas las etapas se operaron a $37^{\circ} \mathrm{C}$. Durante la etapa II, III y IV los reactores fueron adicionados con $10 \mathrm{~g} / \mathrm{L}$ de $\mathrm{NaCl}(1 \%)$. En la etapa $\mathrm{V}$ se adicionaron $30 \mathrm{~g} / \mathrm{L}$ de $\mathrm{NaCl}(3 \%)$. Se tomaron alícuotas tanto de la alimentación como del efluente a lo largo de cada una de las etapas para evaluar las eficiencias de ambos reactores en términos de consumo de glucosa y de decoloración del RC.

\subsection{Métodos de análisis}

Para determinar el contenido de SSV presente en el lodo anaerobio se utilizó el procedimiento indicado en estándar métodos (APHA, 2005). Para la medición de la glucosa se tomaron alícuotas de $1 \mathrm{~mL}$ de ambos reactores cada $24 \mathrm{~h}$, se centrifugaron a $150 \mathrm{rpm}$ por 6 min para eliminar restos de lodo, y el sobrenadante se diluyó con agua destilada para asegurar tener una absorbancia menor a 1 durante la determinación espectrofotométrica. La muestra diluida se preparó para ser analizada mediante el método de fenol-ácido sulfúrico para carbohidratos totales (Nielsen, 2010). Este método consiste en hacer reaccionar $2 \mathrm{~mL}$ de muestra con $50 \mu \mathrm{L}$ de fenol al $80 \%$ y $5 \mathrm{~mL}$ de $\mathrm{H}_{2} \mathrm{SO}_{4}$ durante 10 minutos. Finalmente, las muestras fueron medidas en un espectrofotómetro a $490 \mathrm{~nm}$. Para el análisis del $\mathrm{RC}$ el procedimiento consistió en tomar alícuotas de $1 \mathrm{~mL}$ cada $24 \mathrm{~h}$ y centrifugarlas a $150 \mathrm{rpm}$ por 6 minutos, posteriormente el sobrenadante se diluyó en un buffer de fosfatos (10.86 g/L NaH $2 \mathrm{PO}_{4} \cdot 2 \mathrm{H}_{2} \mathrm{O} ; 5.38 \mathrm{~g} / \mathrm{L} \mathrm{Na} \mathrm{HPO}_{4} \cdot \mathrm{H}_{2} \mathrm{O}$ ) para lograr una absorbancia menor a 1 . Las muestras se midieron en un espectrofotómetro a $485 \mathrm{~nm}$. 


\section{RESULTADOS}

\subsection{Capacidad de inmovilización de AQS en el carbón activado}

La capacidad de adsorción de AQS sobre el CAG fue de $0.588 \mathrm{mmol} / \mathrm{g}$ (Fig. 1). El material modificado (CA-AQS) fue sometido a cinco ciclos de lavado con el medio basal descrito en la sección 2.1, ocasionando una disminución de la capacidad de adsorción a $0.536 \mathrm{mmol} / \mathrm{g}$, que representa la desorción de $8.8 \%$ de AQS. En estudios previos se utilizó CAG del mismo lote de origen, sin embargo, no fue expuesto a la solución de $\mathrm{ZnCl} / 2 / \mathrm{HCl}$. En ese estudio se logró una capacidad de adsorción de $0.276 \mathrm{mmol} / \mathrm{g}$, que representa 2.1 veces menos la cantidad de AQS inmovilizada respecto al valor alcanzado con el CAG-Cl en el presente estudio. Además, ese material fue sometido a seis ciclos de desorción, disminuyendo 18\% la capacidad de adsorción (Alvarez et al., 2017b). Estos resultados indican que el método de modificación del CAG mediante el reactivo de Lucas resultó relevante, considerando que hubo una capacidad de adsorción más alta y un menor desprendimiento de AQS.

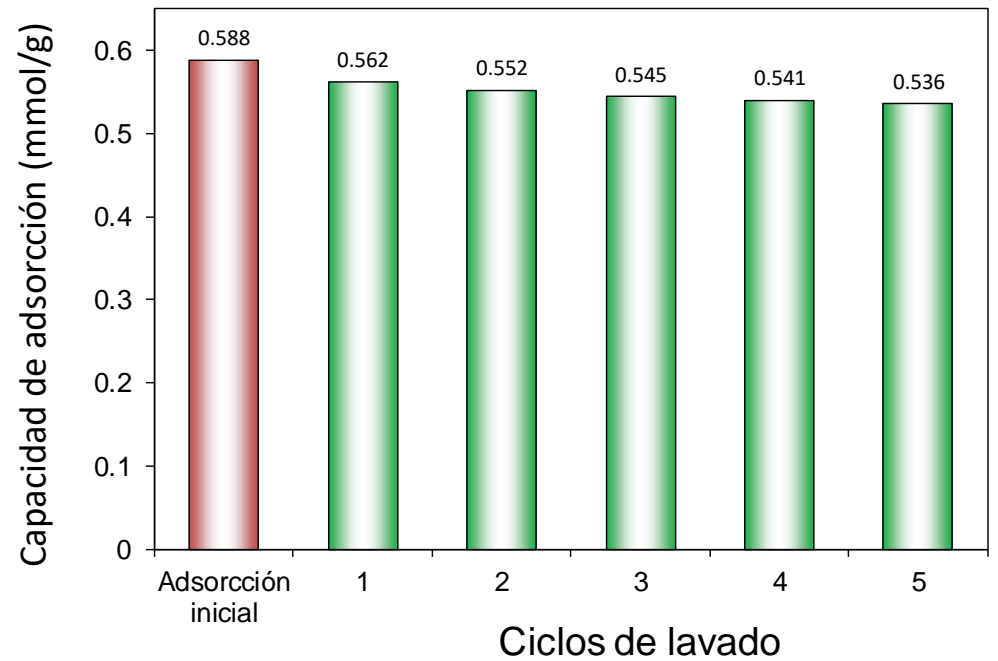

Fig. 1. Capacidad de adsorción de AQS sobre el CAG antes y después de ser sometido a cinco ciclos de lavado con medio basal.

Fig. 1. Adsorption capacity of AQS on GAC before and after of five desorption cycles with basal medium. 

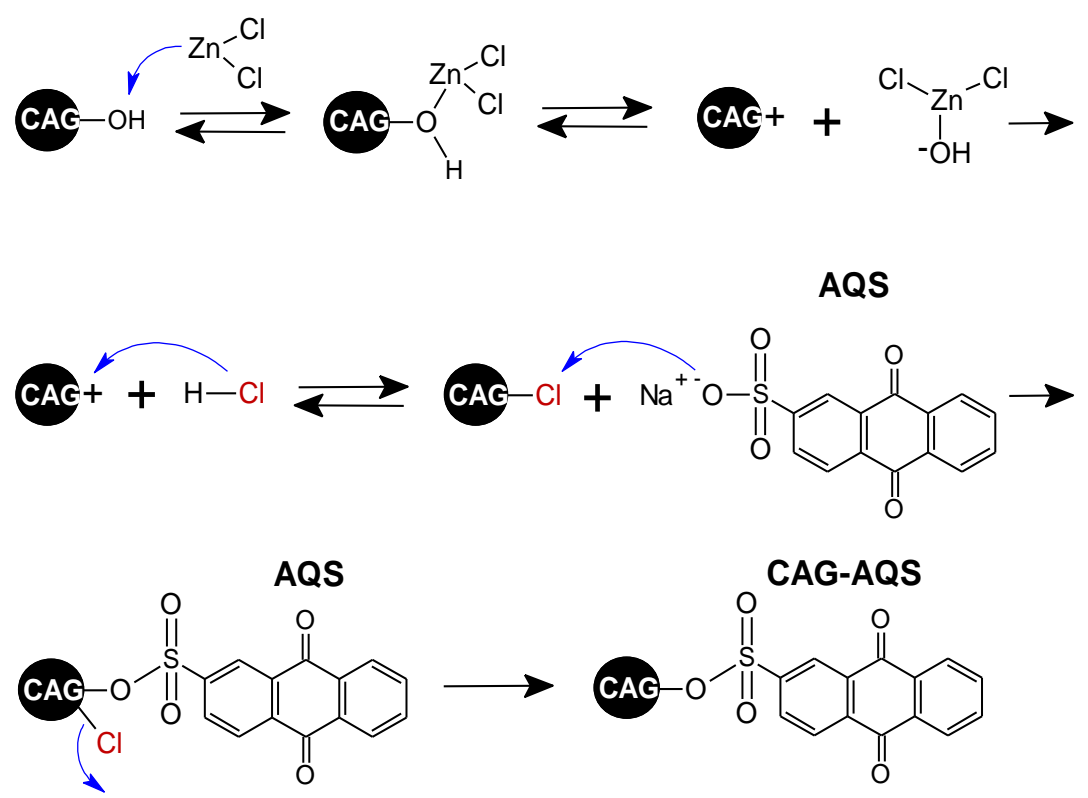

Fig. 2. Mecanismo propuesto para la funcionalización del CAG con AQS utilizando el reactivo de Lucas $\left(\mathrm{HCl} / \mathrm{ZnCl}_{2}\right)$.

Fig. 2. Proposed mechanism for the functionalization of GAC with AQS using the Lucas reagent $\left(\mathrm{HCl} / \mathrm{ZnCl}_{2}\right)$.

El mecanismo de funcionalización del CAG con AQS implica el intercambio de los grupos hidroxilo del CAG por grupos cloruro del ácido clorhídrico. Este paso de adición inicial de grupos cloruro es importante y necesario para llevar a cabo su posterior sustitución por la molécula de AQS a través del anclaje con el grupo sulfónico (Fig. 2). La inmovilización de AQS sobre CAG-Cl puede atribuirse a sitios específicos de adsorción, en donde ocurre una sustitución nucelofílica entre el grupo sulfónico del AQS y un grupo fácilmente saliente como el cloruro presente en el CAG-Cl.

\subsection{Decoloración de rojo congo utilizando AQS inmovilizada}

El reactor CAG-AQS logró un mejor desempeño durante todas las etapas (I-V) en términos de eficiencia de decoloración de RC comparado con el reactor CAG, indicando que la AQS inmovilizada se mantuvo catalíticamente activa durante el periodo de operación (Fig. 3). Durante la etapa I el reactor CAG-AQS alcanzó una eficiencia de decoloración de $88.9 \%$, que contrastó ampliamente con la eficiencia del reactor CAG, que alcanzó en promedio $59.7 \%$ para la misma etapa (Tabla 1 ). En la etapa II, en donde se adicionó $1 \%$ de $\mathrm{NaCl}$, ambos reactores incrementaron la eficiencia de decoloración respecto a la etapa I, alcanzando valores de $93.6 \%$ para el reactor CAG-AQS y de $84.6 \%$ para el reactor CAG, los cuales tienen diferencia estadística significativa (Tabla 1). 


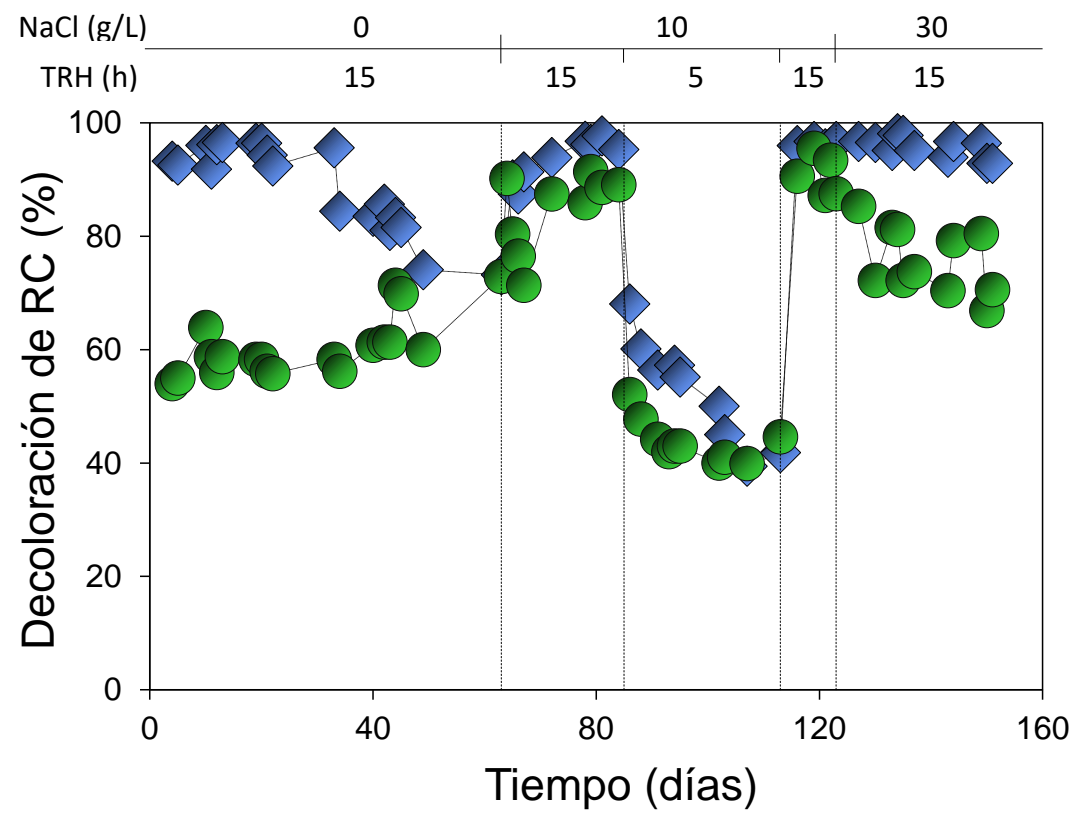

Fig. 3. Eficiencia de decoloración de RC en los reactores UASB CAG-AQS ( $\downarrow$ ) y CAG (•) bajo distintas condiciones de operación.

Fig. 3. Decolorization efficiency of CR in the UASB reactors GAC-AQS ( $\downarrow)$ and GAC $(\bullet)$ under different operational conditions.

Por otro lado, las eficiencias de decoloración de ambos reactores fueron drásticamente afectadas por el cambio de TRH de 15 a $5 \mathrm{~h}$ el iniciar la etapa III. Las eficiencias disminuyeron hasta $52.6 \%$ en el reactor CAG-AQS y $43.8 \%$ para el reactor CAG (Fig. 3). Este decremento en la capacidad de biotransformación de los reactores puede asociarse al aumento de la carga alimentación del RC, que paso de $6.6 \mathrm{mg} / \mathrm{L} \cdot \mathrm{h}$ de la etapa II, a $20 \mathrm{mg} / \mathrm{L} \cdot \mathrm{h}$ en la etapa III. La disminución en la eficiencia de reducción de $\mathrm{RC}$ es comparable con un estudio previo en el que se observó un decremento en la eficiencia de decoloración del colorante naranja ácido 7 a medida que el TRH se disminuyó de $6 \mathrm{~h}$ en donde alcanzó una eficiencia de 95\%, a $2 \mathrm{~h}$, disminuyendo la eficiencia a 70\% (Cervantes et al., 2001). Para la etapa IV, cuando la operación de los reactores volvió al TRH de $15 \mathrm{~h}$, las eficiencias de decoloración se incrementaron a 96.1\% para el reactor CAG-AQS y 90.8\% para el reactor CAG, debido a que la carga de alimentación de RC disminuyó a $6.6 \mathrm{mg} / \mathrm{L} \cdot \mathrm{h}$. En la etapa V, con un aumento en la salinidad a 3\%, los valores de decoloración alcanzaron $95.6 \%$ para el reactor CAG-AQS y $75.8 \%$ para el reactor CAG. Tanto en la etapa IV y $\mathrm{V}$ los valores de decoloración fueron estadísticamente diferentes entre ambos reactores. 


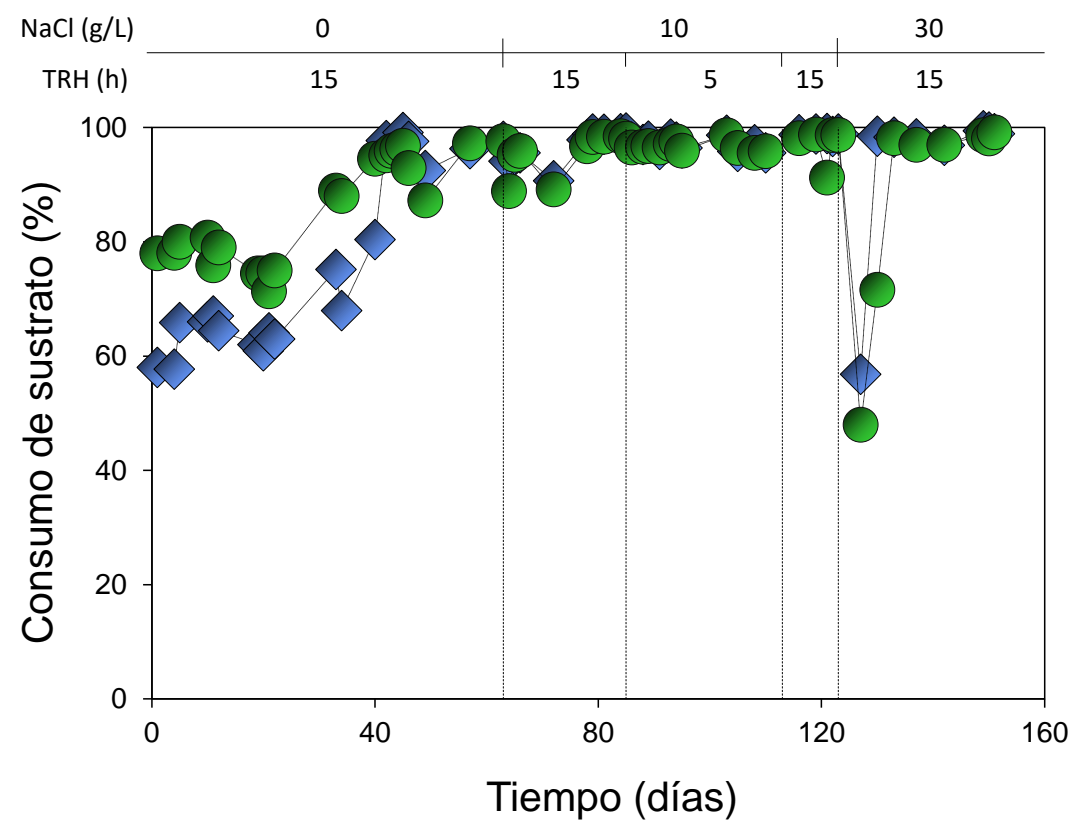

Fig. 4. Eficiencia de consumo de sustrato en los reactores UASB CAG-AQS ( $\bullet$ y CAG $(\bullet)$ bajo distintas condiciones de operación.

Fig. 4. Substrate consumption efficiency in the UASB reactors GAC-AQS ( $\bullet$ ) and GAC $(\bullet)$ under different operational conditions.

El consumo de sustrato durante la etapa I alcanzó los resultados más bajos con valores de $77.4 \%$ y $85.6 \%$ para los reactores CAG-AQS y CAG, respectivamente (Fig. 4). Sin embargo, en la etapa I y en las siguientes, no hay diferencia estadística significativa entre las eficiencias de ambos reactores (Tabla 1). Con la adición de $\mathrm{NaCl}$ a partir de la etapa II, se observó que el consumo de sustrato aumento considerablemente respecto a la etapa I, alcanzando valores superiores a $93 \%$ en las siguientes etapas en ambos reactores, exceptuando en la etapa $\mathrm{V}$ del rector CAG. Contrario al desempeño observado en la etapa III, en donde la eficiencia de decoloración fue afectada por el cambio de TRH de 15 a $5 \mathrm{~h}$, el consumo de sustrato en ambos reactores no se afectó (Tabla 1). 
Tabla 1. Eficiencia de decoloración de $R C(100 \mathrm{mg} / \mathrm{L})$ y de consumo de sustrato (glucosa $1 \mathrm{~g} / \mathrm{L})$ en los reactores UASB con y sin AQS inmovilizada.

Table 1. Decolorization of CR (100 mg/L) and substrate consumption (glucose $1 \mathrm{~g} / \mathrm{L}$ ) efficiencies in the UASB reactor with and without immobilized AQS.

\begin{tabular}{|c|c|c|c|c|c|c|c|}
\hline \multirow{2}{*}{ Etapa } & \multirow{2}{*}{$\begin{array}{l}\text { Periodo } \\
\text { (días) }\end{array}$} & \multirow{2}{*}{$\begin{array}{c}\mathrm{NaCl} \\
(\%)\end{array}$} & \multirow{2}{*}{$\begin{array}{c}\text { TRH } \\
\text { (h) }\end{array}$} & \multicolumn{2}{|c|}{$\begin{array}{c}\text { Decoloración } \\
\text { del RC (\%) }\end{array}$} & \multicolumn{2}{|c|}{$\begin{array}{c}\text { Consumo } \\
\text { de sustrato (\%) }\end{array}$} \\
\hline & & & & CAG-AQS & CAG & CAG-AQS & CAG \\
\hline I & $0-63$ & 0 & 15 & $88.9 \pm 7.7 \mathbf{a}$ & $59.7 \pm 4.7 b$ & $77.4 \pm 6.2 \mathbf{a}$ & $85.6 \pm 9.3 \mathbf{a}$ \\
\hline II & $64-85$ & 1 & 15 & $93.6 \pm 3.6 \mathbf{a}$ & $84.6 \pm 6.9 \mathbf{b}$ & $96.6 \pm 2.9 \mathbf{a}$ & $95.5 \pm 3.8 \mathbf{a}$ \\
\hline III & $86-113$ & 1 & 5 & $52.6 \pm 9.3 \mathbf{a}$ & $43.8 \pm 3.8 b$ & $97.0 \pm 0.9 \mathbf{a}$ & $96.7 \pm 0.9 \mathbf{a}$ \\
\hline IV & $114-123$ & 1 & 15 & $96.1 \pm 0.6 \mathbf{a}$ & $90.8 \pm 3.6 \mathbf{b}$ & $98.8 \pm 0.2 \mathbf{a}$ & $97.1 \pm 3.3 \mathbf{a}$ \\
\hline V & $124-151$ & 3 & 15 & $95.6 \pm 1.8 \mathbf{a}$ & $75.8 \pm 5.9 \mathbf{b}$ & $93.3 \pm 15 \mathbf{a}$ & $88.4 \pm 17 \mathbf{a}$ \\
\hline \multicolumn{8}{|c|}{$\begin{array}{l}\text { Notas: } \\
\text { - Los valores de decoloración de RC y consumo de sustrato representan el promedio de las mediciones del } \\
\text { periodo en cada etapa } \pm \text { la desviación estándar. } \\
\text { - Letras diferentes después de cada valor indican diferencia significativa en las medias }(\mathrm{P}<0.05) \text {. EI } \\
\text { análisis se hizo comparando las medias entre CAG-AQS y CAG para cada etapa. } \\
\text { - Aunque no se indica en la tabla, se analizaron las medias de las eficiencias de decoloración entre las } \\
\text { etapas del mismo reactor, encontrándose que el desempeño del reactor CAG-AQS en la etapa III es } \\
\text { estadísticamente diferente al resto de las etapas. Para el reactor CAG, las etapas II y IV son } \\
\text { estadísticamente iguales y diferentes al resto de las etapas (I, III y V, que son diferentes entre sí). }\end{array}$} \\
\hline
\end{tabular}

\section{DISCUSIÓN}

El tratamiento de aguas residuales textiles ha sido estudiado desde hace muchos años; sin embargo, una de las condiciones que debe ser explorada con mayor detalle es el efecto de la salinidad sobre los sistemas de tratamiento biológico. Se han reportado concentraciones de salinidad de 3\% hasta 20\% (Fernando et al., 2013). La presencia iones de sodio puede impactar de manera importante sobre el grado de biodegradación de colorantes azo, debido a que pueden causar plasmólisis en las células bacterianas (Guo et al., 2005; Manu \& Chaudhari, 2003). Diversos estudios señalan que los tratamientos de decoloración ocurren favorablemente a bajas concentraciones de salinidad (menores a $5 \mathrm{~g} / \mathrm{L}$ ) (Oturkar et al., 2011). En condiciones con concentraciones superiores a $3-5 \%$ de $\mathrm{NaCl}$ hubo inhibición del crecimiento bacteriano e inhibición en la eficiencia de decoloración (Xiao \& Roberts, 2010). Sin embargo, estos estudios fueron conducidos en reactores en lote, a diferencia de los experimentos de este estudio utilizando reactores en continuo y bajo diferentes concentraciones de salinidad. Resultados previos indican que la capacidad de los lodos anaerobios que no han sido adaptados a condiciones salinas tiende a ser inhibida, por lo que se espera que el porcentaje de decoloración sufra un decremento (Guo et al., 2005). Sin embargo, 
en otros estudios se ha encontrado que condiciones de salinidad de hasta $4 \%$ incrementan la velocidad de decoloración de los microorganismos mediada con AQDS (Alvarez et al., 2016), que puede estar asociado a la capacidad enzimática para no sólo tolerar, sino promover su actividad en presencia de $\mathrm{NaCl}$, que puede estar a asociada a la presencia de bacterias halotolerantes (Baxter \& Gibbons, 1956). La etapa III, en donde la concentración de $\mathrm{NaCl}$ es $1 \%$, representó un cambio drástico para ambos reactores debido a la disminución del TRH de 15 a 5 $h$, que fue evidencio por una baja eficiencia de decoloración para el reactor CAGAQS (52.6\%). Aun así, este resultado fue similar a la decoloración de rojo reactivo $2(54.4 \%)$ y superior a la decoloración del naranja ácido 7 (29.2\%), en donde se utilizó un consorcio anaerobio aclimatado durante varios meses a condiciones salinas (Yuan et al., 2012), a diferencia del lodo en este estudio que no fue adaptado previamente a esas condiciones. Cuando el $\mathrm{TRH}$ cambio para volver nuevamente a $15 \mathrm{~h}$ a partir de la etapa IV, ambos reactores recuperaron su desempeño para biotransformar el RC; sin embargo, el reactor con CAG-AQS tuvo un mejor desempeño que el reactor CAG.

La mayoría de los estudios en los que se ha reportado el uso de MR en procesos de biotransformación de contaminantes han sido conducidos en reactores en lote, que si bien es cierto brindan información valiosa, la aplicación en continuo brinda información para aplicaciones en escenario reales. Además, también son pocos los estudios que involucran MR y condiciones de salinidad de manera simultánea. Algunos estudios en sistemas en lotes reportan inhibición de la decoloración cuando la concentración salina cambia de 1\% a 2.5\% (Fernando et al., 2013). También se ha reportado la inhibición de la actividad debido a una exposición continua a concentraciones mayores al 2\% (Lefebvre \& Moletta, 2006). Por otro lado, en un estudio para tratar aguas residuales que contenían rojo reactivo brillante K-2BP se encontró que conforme se incrementó la concentración de salinidad, disminuyó la eficiencia de decoloración del consorcio halotolerante (Guo et al., 2005), por lo que en estudios posteriores se empleó AQS inmovilizado en perlas de alginato de calcio como MR (Guo et al., 2007). Los resultados obtenidos con el AQS inmovilizado en perlas de alginato indican que se logró decolorar distintos colorantes azo a una concentración de salinidad de hasta 15\%, sin embargo, la resistencia mecánica de las perlas disminuyó gradualmente hasta perder su capacidad en pocos días. Por el contario, el CAG-AQS utilizado en este estudio mantuvo la capacidad catalítica para biotransformar eficientemente el RC durante 150 días bajo condiciones de salinidad de 1\% 0 3\% y diferentes $\mathrm{TRH}$, incluso por encima de los valores del reactor control (Tabla 1).

Durante el periodo $\mathrm{V}$ (3\% de salinidad), el reactor CAG-AQS logró mantener estable y en valores altos la eficiencia de decoloración, incluso bajo concentraciones de salinidad que han sido reportadas como altas tanto en sistema continuos como en lote, para lodos anaerobios no adaptados a salinidad (Aslan \& Şekerdağ, 2015; Guo et al., 2005). En este periodo, ambos reactores tuvieron la capacidad de llevar a cabo de la decoloración de RC, aunque con resultados 
distintos (Fig. 3). Esto indica que incluso bajo altas concentraciones de salinidad el lodo anaerobio se mantuvo metabólicamente activo. Sin embargo, la diferencia en la eficiencia de decoloración indica que el lodo se comportó de manera distinta en ambos reactores, siendo 1.26 veces mayor en el reactor CAG-AQS comparado con el reactor CAG, debido a la adición del MR inmovilizado. Los resultados de las etapas IV y V son consistentes con estudios que reportan un incremento en la decoloración a TRH mas altos, debido a que existe un contacto prolongado entre el consorcio anaerobio y el colorante (Alvarez et al. 2017a; Isik \& Sponza, 2005). Además, la eficiencia de consumo de sustrato fue mayor a $88 \%$ para ambos reactores durante las dos etapas (IV y V), evidenciando que el lodo anaerobio no se afectó por la bencidina producida debido a la ruptura de los enlaces tipo azo del RC (Pereira et al., 2016).

Durante los periodos en los que se evaluó la adición de $\mathrm{NaCl}$ al medio de alimentación, ambos reactores presentaron una eficiencia de consumo de sustrato similar. La adición de $1 \%$ de $\mathrm{NaCl}$ durante los periodos II, III y IV generó eficiencias de consumo de sustrato superiores a 95\% en ambos reactores (Tabla 1), indicando un desempeño adecuado del consorcio bacteriano. En un estudio previo se alcanzó una eficiencia de consumo de sustrato de $75 \%$ durante el tratamiento de aguas residuales que contenían menos de $2 \%$ de $\mathrm{NaCl}$ (Abou-Elela et al., 2010; Lefebvre et al., 2007). No todos los procesos biológicos se desempeñan de manera similar en presencia de $\mathrm{NaCl}$. Por ejemplo, se ha reportado que el consumo de sustrato disminuyó de manera importante con salinidad por encima del 1\% (Aslan \& Şekerdağ, 2015). Sin embargo, tanto en el reactor CAG-AQS como en el CAG, la adición de 1\% de salinidad estimuló el consumo de sustrato. Al aumentar a $3 \%$ de $\mathrm{NaCl}$ durante el periodo $\mathrm{V}$ hubo una disminución en la eficiencia de consumo de sustrato para ambos reactores, siendo más afacetado el reactor sin AQS (Fig. 4). Los resultados son comparables con un estudio en donde el aumento a $3 \%$ de $\mathrm{NaCl}$ generó una disminución en el consumo de sustrato de $94 \%$ a $64 \%$; sin embargo, el lodo empleado no logró recuperar su eficiencia de consumo de sustrato (Abou-Elela et al., 2010). En tanto, en el presente estudio, el reactor CAG-AQS logró 93.3\% de consumo de sustrato, asimismo, se observó que al reactor CAG le tomó mayor tiempo recuperarse ante el cambio de condiciones. El promedio de consumo de sustrato para ambos reactores (CAG-AQS 93.3\% y CAG 88.44\%) resultó muy por encima del reportado para un reactor UASB, en el cual la eficiencia de consumo de sustrato fue $80.5 \%$ con $3 \%$ de $\mathrm{NaCl}$ (Isik, 2004).

La inmovilización de AQS sobre el CAG permitió obtener un material capaz de utilizarse para mejorar la reducción de $\mathrm{RC}$ y el consumo de sustrato bajo las concentraciones de salinidad y condiciones de TRH probadas. El reactor CAGAQS mostró altas eficiencias de decoloración y consumo de sustrato, incluso bajo la concentración de $3 \%$ de salinidad, alcanzando $95.6 \%$ y 93.3\%, respectivamente; en tanto el reactor CAG logró $75.8 \%$ y $88.4 \%$, para esos mismos parámetros. En este estudio se presenta una alternativa para tratar aguas 
residuales textiles que contienen colorantes azo y altos niveles de salinidad. Aunque se logró la decoloración del colorante RC, se recomienda un tratamiento posterior para llevar a cabo la mineralización de las aminas aromáticas que se producen típicamente bajo condiciones de reducción anaerobia. Además, es necesario probar este tipo de sistemas para tratar una mezcla de colorantes e incluso utilizar efluentes de aguas residuales textiles reales. En cuanto al consorcio anaerobio, resulta interesante someterlo a un periodo de aclimatación en condiciones salinas para realizar un análisis más completo acerca de cómo cambiaría la cinética de degradación del colorante, incluso con porcentajes de salinidad más altos.

\section{AGRADECIMIENTOS}

Los autores agradecen el apoyo otorgado por la Facultad de Ciencias Químicas y la Facultad de Ciencias Biológicas de la Universidad Autónoma de Nuevo León. También al Instituto Tecnológico de Sonora por el apoyo a través del Programa de Fomento y Apoyo a Proyectos de Investigación (PROFAPI-ITSON, 2021).

\section{CONFLICTO DE INTERESES}

Los autores declaran no tener ningún conflicto de intereses.

\section{REFERENCIAS}

Abou-Elela S. I., Kamel M. M. \& Fawzy M. E. 2010. Biological treatment of saline wastewater using a salt-tolerant microorganism. Desalination. 250(1): 1-5. https://doi.org/10.1016/i.desal.2009.03.022.

Alvarez L. H., Arvizu I. C., García-Reyes R. B., Martínez C. M., Olivo-Alanís D. \& Del Angel Y. A. 2017a. Quinone-functionalized activated carbon improves the reduction of congo red coupled to the removal of $p$-cresol in a UASB reactor. Journal of Hazardous Materials. 338: 233-240. https://doi.org/10.1016/i.jhazmat.2017.05.032.

Alvarez L. H., Del Angel Y. A. \& García-Reyes B. 2017b. Improved microbial and chemical reduction of direct blue 71 using anthraquinone-2, 6-disulfonate immobilized on granular activated carbon. Water, Air, \& Soil Pollution. 228(1): 38. https://doi.org/10.1007/s11270-016-3212-5.

Alvarez L. H., Jímenez B. L., Hernandez-Montoya V. \& Cervantes F. J. 2012. Enhanced dechlorination of carbon tetrachloride by immobilized fulvic acids on alumina particles. Water, Air, \& Soil Pollution. 223(4): 1911-1920. https://doi.org/10.1007/s11270-011-0994-3.

Alvarez L. H., Meza-Escalante E. R., Gortáres-Moroyoqui P., Morales L., Rosas K., García-Reyes B. \& García-González A. 2016. Influence of redox mediators and 
salinity level on the (bio)transformation of direct blue 71: kinetics aspects. Journal of Environmental Management. 183: 84-89. https://doi.org/10.1016/j.jenvman.2016.08.044.

Alvarez L. H., Pérez-Cruz M. A., Rangel-Méndez J. R. \& Cervantes F. J. 2010. Immobilized redox mediator on metal-oxides nanoparticles and its catalytic effect in a reductive decolorization process. Journal of Hazardous Materials. 184(1-3): 268272. https://doi.org/10.1016/j.jhazmat.2010.08.032.

Amezquita-Garcia H. J., Rangel-Méndez J. R., Cervantes F. J. \& Razo-Flores E. 2016. Activated carbon fibers with redox-active functionalities improves the continuous anaerobic biotransformation of 4-nitrophenol. Chemical Engineering Journal. 286: 208-215. https://doi.org/10.1016/j.cej.2015.10.085.

APHA. (2005). Standard methods for the examination of water and wastewater. American Public Health Association (APHA): Washington, DC, USA.

Aslan S. \& Şekerdağ N. 2016. Salt inhibition on anaerobic treatment of high salinity wastewater by upflow anaerobic sludge blanket (UASB) reactor. Desalination and Water Treatment. 57(28): 12998-13004. https://doi.org/10.1080/19443994.2015.1059369.

Baxter R. M. \& Gibbons N. E. 1956. Effects of sodium and potassium chloride on certain enzymes of Micrococcus halodenitrificans and Pseudomonas salinaria. Canadian Journal of Microbiology. 2(6): 599-606. https://doi.org/10.1139/m56-072.

Brown D. \& Laboureur P. 1983. The aerobic biodegradability of primary aromatic amines. Chemosphere. 12(3): 405-414. https://doi.org/10.1016/00456535(83)90115-7.

Castañon D., Alvarez L. H., Peña K., García-Reyes R. B., Martínez C. M. \& PatEspadas A. 2019. Azo dye biotransformation mediated by AQS immobilized on activated carbon cloth in the presence of microbial inhibitors. Environmental Pollution. 252: 1163-1169. https://doi.org/10.1016/i.envpol.2019.06.050.

Cervantes F. J., García-Espinosa A., Moreno-Reynosa M. A. \& Rangel-Méndez J. R. 2010. Immobilized redox mediators on anion exchange resins and their role on the reductive decolorization of azo dyes. Environmental Science \& Technology. 44(5): 1747-1753. https://doi.org/10.1021/es9027919.

Cervantes F. J., Gonzalez-Estrella J., Márquez A., Alvarez L. H. \& Arriaga S. 2011. Immobilized humic substances on an anion exchange resin and their role on the redox biotransformation of contaminants. Bioresource Technology. 102(2): 20972100. https://doi.org/10.1016/j.biortech.2010.08.021.

Cervantes F. J., Van Der Zee F. P., Lettinga G. \& Field J. A. 2001. Enhanced decolourisation of acid orange 7 in a continuous UASB reactor with quinones as 
redox mediators. Water Science and Technology. 44(4): 123-128. https://doi.org/10.2166/wst.2001.0198.

Fernando E., Keshavarz T. \& Kyazze G. 2013. Simultaneous co-metabolic decolourisation of azo dye mixtures and bio-electricity generation under thermophillic $\left(50^{\circ} \mathrm{C}\right)$ and saline conditions by an adapted anaerobic mixed culture in microbial fuel cells. Bioresource Technology. 127: 1-8. https://doi.org/10.1016/i.biortech.2012.09.065.

Guo J., Zhou J., Wang D., Yang J. \& Li Z. 2008. The new incorporation biotreatment technology of bromoamine acid and azo dyes wastewaters under highsalt conditions. Biodegradation. 19(1): 93-98. https://doi.org/10.1007/s10532-0079118-6.

Guo J., Zhou J., Wang D., Tian C., Wang P., Uddin M. S. \& Yu H. 2007. Biocalalyst effects of immobilized anthraquinone on the anaerobic reduction of azo dyes by the salt-tolerant bacteria. Water Research. 41(2): 426-432. https://doi.org/10.1016/j.watres.2006.10.022.

Guo J. B., Zhou J. T., Wang D., Wang J., Yu H. \& Song Z. Y. 2005. Decolorization of azo dyes with high salt concentration by salt-tolerant mixed cultures under anaerobic conditions. Journal of Environmental Sciences. 17(6): 984-988. https://pubmed.ncbi.nlm.nih.gov/16465892/.

Huang J., Wu M., Chen J., Liu X., Chen T., Wen Y. \& Xie Z. 2015. Enhanced azo dye removal in a continuously operated up-flow anaerobic filter packed with henna plant biomass. Journal of Hazardous Materials. 299: 158-164. https://doi.org/10.1016/i.jhazmat.2015.05.044.

Işik M. 2004. Efficiency of simulated textile wastewater decolorization process based on the methanogenic activity of upflow anaerobic sludge blanket reactor in salt inhibition condition. Enzyme and Microbial Technology. 35(5): 399-404. https://doi.org/10.1016/i.enzmictec.2004.04.018.

Işık M. \& Sponza D. T. 2005. Effects of alkalinity and co-substrate on the performance of an upflow anaerobic sludge blanket (UASB) reactor through decolorization of congo red azo dye. Bioresource Technology, 96(5): 633-643. https://doi.org/10.1016/j.biortech.2004.06.004.

Lefebvre O. \& Moletta R. 2006. Treatment of organic pollution in industrial saline wastewater: a literature review. Water Research. 40(20): 3671-3682. https://doi.org/10.1016/i.watres.2006.08.027.

Lefebvre O., Quentin S., Torrijos M., Godon J. J., Delgenes J. P. \& Moletta R. 2007. Impact of increasing $\mathrm{NaCl}$ concentrations on the performance and community composition of two anaerobic reactors. Applied Microbiology and Biotechnology. 75(1): 61-69. https://doi.org/10.1007/s00253-006-0799-2. 
Li L., Wang J., Zhou J., Yang F., Jin C., Qu Y. \& Zhang L. 2008. Enhancement of nitroaromatic compounds anaerobic biotransformation using a novel immobilized redox mediator prepared by electropolymerization. Bioresource Technology. 99(15): 6908-6916. https://doi.org/10.1016/j.biortech.2008.01.037.

Li L., Zhou J., Wang J., Yang F., Jin C. \& Zhang G. 2009. Anaerobic biotransformation of azo dye using polypyrrole/anthraquinonedisulphonate modified active carbon felt as a novel immobilized redox mediator. Separation and $\begin{array}{llll}\text { Purification } & \text { Technology. 66(2): 375-382. }\end{array}$ https://doi.org/10.1016/j.seppur.2008.12.019.

Lu H., Zhou J., Wang J., Si W., Teng H. \& Liu G. 2010. Enhanced biodecolorization of azo dyes by anthraquinone-2-sulfonate immobilized covalently in polyurethane foam. Bioresource Technology. 101(18): 7185-7188. https://doi.org/10.1016/j.biortech.2010.04.007.

Manu B. \& Chaudhari S. 2003. Decolorization of indigo and azo dyes in semicontinuous reactors with long hydraulic retention time. Process Biochemistry 38(8): 1213-1221. https://doi.org/10.1016/S0032-9592(02)00291-1.

Martínez C., Díaz R., Sánchez L. \& Díaz G. 2018. Biodegradation of azo dyes by Pleurotus ostreatus. Mexican Journal of Biotechnology. 3(1): 43-59. 30. https://doi.org/10.29267/mxib.2018.3.1.43.

Martínez C. M., Alvarez L. H., Celis L. B. \& Cervantes F. J. 2013. Humus-reducing microorganisms and their valuable contribution in environmental processes. Applied Microbiology and Biotechnology. 97(24): 10293-10308. https://doi.org/10.1007/s00253-013-5350-7.

Nielsen S. S. 2010. Phenol-sulfuric acid method for total carbohydrates. In Food analysis laboratory manual (pp. 47-53). Springer, Boston, MA. https://doi.org/10.1007/978-1-4419-1463-7 6.

O’Neill C., Hawkes F. R., Hawkes D. L., Lourenço N. D., Pinheiro H. M. \& Delée W. 1999. Colour in textile effluents-sources, measurement, discharge consents and simulation: a review. Journal of Chemical Technology \& Biotechnology: International Research in Process, Environmental \& Clean Technology. 74(11): 1009-1018. $\quad$ https://doi.org/10.1002/(SICI)1097-4660(199911)74:11<1009::AIDJCTB153>3.0.CO;2-N.

Oturkar C. C., Nemade H. N., Mulik P. M., Patole M. S., Hawaldar R. R. \& Gawai K. R. 2011. Mechanistic investigation of decolorization and degradation of Reactive Red 120 by Bacillus lentus B|377. Bioresource Technology. 102(2): 758-764. https://doi.org/10.1016/j.biortech.2010.08.094. 
Pagga U. \& Brown D. 1986. The degradation of dyestuffs: Part II Behaviour of dyestuffs in aerobic biodegradation tests. Chemosphere. 15(4): 479-491. https://doi.org/10.1016/0045-6535(86)90542-4.

Pereira L., Pereira R., Pereira M. F. R., Van Der Zee F. P., Cervantes F. J. \& Alves M. M. 2010. Thermal modification of activated carbon surface chemistry improves its capacity as redox mediator for azo dye reduction. Journal of Hazardous Materials. 183(1-3): 931-939. https://doi.org/10.1016/j.jhazmat.2010.08.005.

Pereira R. A., Salvador A. F., Dias P., Pereira M. F. R., Alves M. M. \& Pereira L. 2016. Perspectives on carbon materials as powerful catalysts in continuous anaerobic bioreactors. Water Research. 101: 441-447. https://doi.org/10.1016/i.watres.2016.06.004.

Rodríguez S. Y., Cantú M. E., Garcia-Reyes B., Garza-Gonzalez M. T., MezaEscalante E. R., Serrano D. \& Alvarez L. H. 2019. Biotransformation of 4nitrophenol by co-immobilized Geobacter sulfurreducens and anthraquinone-2sulfonate in barium alginate beads. Chemosphere. 221: 219-225. https://doi.org/10.1016/j.chemosphere.2019.01.041.

Talarposhti A. M., Donnelly T. \& Anderson G. K. 2001. Colour removal from a simulated dye wastewater using a two-phase anaerobic packed bed reactor. Water Research. 35(2): 425-432. https://doi.org/10.1016/S0043-1354(00)00280-3.

Van Der Zee F. P. \& Cervantes F. J. 2009. Impact and application of electron shuttles on the redox (bio) transformation of contaminants: a review. Biotechnology Advances. 27(3): 256-277. https://doi.org/10.1016/j.biotechadv.2009.01.004.

Van Der Zee F. P., Bisschops I. A., Lettinga G. \& Field J. A. (2003). Activated carbon as an electron acceptor and redox mediator during the anaerobic biotransformation of azo dyes. Environmental Science \& Technology, 37(2): 402408. https://doi.org/10.1021/es0258850.

Xiao Y. \& Roberts D. J. 2010. A review of anaerobic treatment of saline wastewater. Environmental Technology. 31(8-9): 1025-1043. https://doi.org/10.1080/09593331003734202.

Yuan S. Z., Lu H., Wang J., Zhou J. T., Wang Y. \& Liu G. F. 2012. Enhanced biodecolorization of azo dyes by quinone-functionalized ceramsites under saline conditions. $\quad$ Process Biochemistry. $47(2)$ : 312-318. https://doi.org/10.1016/..procbio.2011.11.015.

Zhang D., Zhang C., Li Z., Suzuki D., Komatsu D. D., Tsunogai U. \& Katayama A. 2014. Electrochemical stimulation of microbial reductive dechlorination of pentachlorophenol using solid-state redox mediator (humin) immobilization. Bioresource Technology. 164: 232-240. https://doi.org/10.1016/j.biortech.2014.04.071. 